PROCEEDINGS OF THE

AMERICAN MATHEMATICAL SOCIETY

Volume 137, Number 5, May 2009, Pages 1753-1761

S 0002-9939(08)09678-0

Article electronically published on November 17, 2008

\title{
DISTINGUISHING PROPERTIES OF ARENS IRREGULARITY
}

\author{
ZHIGUO HU AND MATTHIAS NEUFANG
}

(Communicated by Nigel J. Kalton)

\begin{abstract}
In this paper, we present a number of examples of commutative Banach algebras with various Arens irregularity properties. These examples illustrate in particular that strong Arens irregularity and extreme non-Arens regularity, the two natural concepts of "maximal" Arens irregularity for general Banach algebras as introduced by Dales-Lau and Granirer, respectively, are indeed distinct. Thereby, an open question raised by several authors is answered. We also link these two properties to another natural Arens irregularity property.
\end{abstract}

\section{INTRODUCTION}

Let $A$ be a Banach algebra. As is well-known, on the bidual $A^{* *}$ of $A$, there are two Banach algebra multiplications, called the left and right Arens products, each extending the multiplication on $A$ (see [1]). By definition, the left Arens product $\square$ on $A^{* *}$ is induced by the left $A$-module structure on $A$. That is, for $m, n \in A^{* *}$, $f \in A^{*}$, and $a, b \in A$, we have

$$
\langle m \square n, f\rangle=\langle m, n \square f\rangle,\langle n \square f, a\rangle=\langle n, f \cdot a\rangle \text {, and }\langle f \cdot a, b\rangle=\langle f, a b\rangle .
$$

Similarly, the right Arens product $\diamond$ on $A^{* *}$ is defined by considering $A$ as a right $A$-module. $A$ is said to be Arens regular if $\square$ and $\diamond$ coincide on $A^{* *}$. Every $C^{*}$ algebra is Arens regular. On the other hand, Banach algebras studied in abstract harmonic analysis are typically far from being Arens regular.

By definition, for any fixed $m \in A^{* *}$, the maps $n \longmapsto n \square m$ and $n \longmapsto m \diamond n$ are weak*-weak* continuous on $A^{* *}$. The left and the right topological centres of $A^{* *}$ are defined, respectively, as

$Z_{t}^{(l)}\left(A^{* *}\right)=\left\{m \in A^{* *}:\right.$ the map $n \longmapsto m \square n$ is weak*-weak ${ }^{*}$ continuous on $\left.A^{* *}\right\}$, $Z_{t}^{(r)}\left(A^{* *}\right)=\left\{m \in A^{* *}:\right.$ the map $n \longmapsto n \diamond m$ is weak ${ }^{*}$-weak ${ }^{*}$ continuous on $\left.A^{* *}\right\}$.

It can be seen that $A$ is Arens regular if and only if $Z_{t}^{(l)}\left(A^{* *}\right)=Z_{t}^{(r)}\left(A^{* *}\right)=A^{* *}$. The reader is referred to Dales [4], Dales-Lau [5], Lau-Ülger [22], and Palmer [26] for more information on Arens products and topological centres.

Received by the editors June 16, 2008.

2000 Mathematics Subject Classification. Primary 43A20, 43A30, 46H05.

Key words and phrases. Banach algebras, Arens products, topological centres, weakly almost periodic functionals, Fourier algebras.

Both authors were partially supported by NSERC. 
Clearly, $A \subseteq Z_{t}^{(l)}\left(A^{* *}\right) \cap Z_{t}^{(r)}\left(A^{* *}\right)$ under the canonical embedding $A \hookrightarrow A^{* *}$. The following concepts of Arens irregularity were introduced by Dales and Lau [5]: $A$ is called left strongly Arens irregular if $Z_{t}^{(l)}\left(A^{* *}\right)=A$, right strongly Arens irregular if $Z_{t}^{(r)}\left(A^{* *}\right)=A$, and strongly Arens irregular (SAI for short) if $A$ is both left and right strongly Arens irregular.

Let $G$ be a locally compact group. It was proved by Lau and Losert [19] that the group algebra $L_{1}(G)$ is always SAI. For the Fourier algebra $A(G)$, Lau and Losert [20. Theorem 6.5] showed that $A(G)$ is SAI if either $G$ is discrete and amenable, or $G$ is second countable and amenable with $\overline{[G, G]}$ non-open in $G$. Moreover, Lau and Losert 21] also proved that $A(G)$ is SAI when $G$ is a countably infinite product of second countable locally compact groups $\left\{G_{i}\right\}_{i=0}^{\infty}$ with $G_{i}(i \geq 1)$ compact and non-trivial. Some slight extensions of [20, Theorem 6.5(iii)] and [21, Theorem 4.2] were obtained by the first author in 15 . However, as shown recently by Losert in 24] and [25], respectively, $A\left(F_{2}\right)$ and $A(S U(3))$ are not SAI.

There is another notion of Arens irregularity for a general Banach algebra $A$ in the literature. Let $W A P(A)$ be the space of weakly almost periodic functionals on $A$. It was shown by $\mathrm{Pym}\left[27\right.$ that $A$ is Arens regular if and only if $W A P(A)=A^{*}$. Hence, the size of the quotient space $A^{*} / W A P(A)$ measures the Arens irregularity of $A$ in a certain sense. In 11, Granirer introduced the concept of "extreme nonArens regularity": $A$ is called extremely non-Arens regular (ENAR for short) if $A^{*} / W A P(A)$ contains a closed linear subspace which has $A^{*}$ as a continuous linear image.

Corollary 7 in Granirer [11] shows that $A(G)$ is ENAR if $G$ is non-discrete and second countable. The first author extended Granirer's result by proving that $A(G)$ is ENAR if $G$ is infinite and satisfies $\kappa(G) \leq \chi(G)$, where $\kappa(G)$ is the compact covering number of $G$ and $\chi(G)$ is the local weight of $G$ (see [12, Corollary 4.2]). Versions for $L_{1}(G)$ of some results on $A(G)$ by Granirer 11 and the first author 12, 13 were obtained by Fong and the second author in [6], and also recently by Bouziad and Filali in 3. In particular, it was shown that $L_{1}(G)$ is ENAR for all $\sigma$-compact non-compact locally compact groups $G$ (cf. [6, Theorem 4.7] and [3, Theorem 4.4]).

The counterexample $A(S U(3))$ to the strong Arens irregularity problem of Fourier algebras, presented by Losert, gives a Banach algebra which is ENAR but not SAI. The question of whether there exists an SAI and Arens irregular Banach algebra which fails to be ENAR was raised by the authors in [16] (see also Bouziad-Filali [3]).

In this note, we present a number of examples of Arens irregular commutative Banach algebras. These examples illustrate in particular that these two natural concepts of "maximal" Arens irregularity for general Banach algebras are indeed distinct (cf. Theorems 4 and 5). Thereby, the open question on the relation between strong Arens irregularity and extreme non-Arens regularity is answered, as conjectured by the authors in [16. We also link these two properties to yet another Arens irregularity property, which will be introduced in the next section.

\section{BANACH ALGEBRAS WITH WEAK FACTORIZATION}

Let $A$ be a Banach algebra. Let $\left\langle A^{*} A\right\rangle$ and $\left\langle A^{*} A^{* *}\right\rangle$ denote the closed linear spans of the module products $A^{*} \cdot A$ and $A^{*} \diamond A^{* *}$, respectively. Then $\left\langle A^{*} A\right\rangle \subseteq$ $\left\langle A^{*} A^{* *}\right\rangle \subseteq A^{*}$. By Cohen's factorization theorem, $\left\langle A^{*} A\right\rangle=A^{*} \cdot A$ if $A$ has a 
bounded approximate identity (BAI). In this situation, $A^{*}$ is said to factor on the left if $A^{*} \cdot A=A^{*}$ (see Lau-Ülger [22]). In [22, Theorem 2.6] Lau and Ülger proved that if $A$ is weakly sequentially complete with a sequential BAI, then $A^{*}$ factors on the left if and only if $A$ is unital. This result of Lau and Ülger has been extended recently by the authors and Ruan to a larger class of Banach algebras, which includes all convolution Beurling algebras $L_{1}(G, \omega)$ with $\omega \geq 1$ and Fourier algebras $A(G)$ with $G$ amenable (see [17, 18]).

Definition 1. Let $A$ be a Banach algebra and let $X$ be a right Banach $A$-module. $X$ is said to factor over $A$ on the left if $\langle X \cdot A\rangle=X$. When $X=A^{*}$ has its canonical $A$-module structure, the term "over $A$ " is omitted. When $X$ is a symmetric Banach $A$-module, the term "on the left" is omitted.

If $A$ is commutative, $A^{* *} \square A^{*}=A^{*} \diamond A^{* *}$. In this case, we say that $A$ has WF (weak factorization) if $A^{*}$ factors over $A^{* *}$ (i.e., $\left\langle A^{*} A^{* *}\right\rangle=A^{*}$ ), and $A$ has PWF (proper weak factorization) if $A^{*}$ factors over $A^{* *}$ but not over $A$ (i.e., $\left\langle A^{*} A\right\rangle \varsubsetneqq$ $\left.\left\langle A^{*} A^{* *}\right\rangle=A^{*}\right)$.

Let $G$ be a locally compact group. If $A=L_{1}(G)$, then $\left\langle A^{*} A\right\rangle$ is the $C^{*}$-algebra $L U C(G)$ of left uniformly continuous functions on $G$; hence $L_{1}(G)^{*}$ factors on the left if and only if $G$ is discrete. If $A=A(G)$, then $\left\langle A^{*} A\right\rangle$ is the $C^{*}$-algebra $U C B(\widehat{G})$ of uniformly continuous linear functionals on $A(G)$ (see Granirer [9]). By [10, Theorem 3], $A(G)^{*}$ factors if and only if $G$ is compact.

In the rest of this section, $A$ will denote a commutative Banach algebra with a faithful multiplication. In this situation, $Z_{t}^{(l)}\left(A^{* *}\right)=Z_{t}^{(r)}\left(A^{* *}\right)$ is just the algebraic centre $Z\left(A^{* *}\right)$ of $A^{* *}$, and $A^{*}$ is both an $A$-module and an $A^{* *}$-module, where $A^{* *}$ is equipped with either of the Arens products.

Obviously, if $A$ has a BAI, then $\left\langle A^{*} A^{* *}\right\rangle=A^{*}$. Hence, when considering Banach algebras with WF or PWF, we are most interested in Banach algebras which do not have any BAI.

It turns out that the two extreme cases in the inclusions $\left\langle A^{*} A\right\rangle \subseteq\left\langle A^{*} A^{* *}\right\rangle \subseteq A^{*}$ are related to Arens (ir)regularity of $A$. In fact, it was shown by the first author [15, Lemma 2.2(iii)] that for all commutative Banach algebras $A, Z\left(A^{* *}\right) \cap\left\langle A^{*} A\right\rangle^{\perp}=$ $\left\langle A^{*} A^{* *}\right\rangle^{\perp}$. Thus, we have

$$
A \text { is Arens regular } \Longrightarrow\left\langle A^{*} A\right\rangle=\left\langle A^{*} A^{* *}\right\rangle
$$

and

$$
A \text { is } \mathrm{SAI} \Longrightarrow\left\langle A^{*} A^{* *}\right\rangle=A^{*} .
$$

It follows from (1) that

$$
A \text { has } \mathrm{PWF} \Longrightarrow A \text { is Arens irregular and non-unital. }
$$

Therefore, having PWF indeed describes an Arens irregularity property for nonunital commutative Banach algebras. It will be seen in Section 3 that this property is also distinct from strong Arens irregularity and extreme non-Arens regularity.

In general, the converses of (1) and (2) are not true, since if $A$ is unital, then $\left\langle A^{*} A\right\rangle=\left\langle A^{*} A^{* *}\right\rangle=A^{*}$, but $A$ may be neither Arens regular nor SAI. However, if $A$ is semisimple, weakly sequentially complete, and completely continuous with $A^{*}$ a von Neumann algebra, Ülger [28, Theorem 3.3(e)] shows that the converse of (1) holds. Therefore, for all discrete groups $G$,

$$
A(G) \text { is Arens regular } \Longleftrightarrow\left\langle A(G)^{*} A(G)^{* *}\right\rangle=U C B(\widehat{G}) .
$$


In Section 3, we will construct a "non-trivial" counterexample to the converse of (2), i.e., a Banach algebra which does not have a BAI and for which the converse of (2) fails (Theorem 5).

On the other hand, the first author proved that for all locally compact groups $G$, $A(G)$ is SAI if and only if $A(G)$ has WF and $A(H)$ is SAI for every $\sigma$-compact open subgroup $H$ of $G$ (see [15, Theorem 3.9]). For discrete groups $G$ containing the free group $F_{r}$ on $r$ generators with $2 \leq r<\infty$, beyond showing that $A(G)$ is not SAI, Losert [24, Corollary 1] proved that $A(G)$ does not even have WF (cf. (2)). Note that $A\left(F_{2}\right)$ is non-unital and Arens irregular (see e.g. Forrest [7, Corollary 3.8]). Therefore, the converse of (3) does not hold in general.

We close this section by recalling that $U C B(\widehat{G}) \varsubsetneqq A(G) *$ if $G$ is not compact (see Granirer [10, Theorem 3]). Therefore, for all non-compact locally compact groups $G$, we have

$$
A(G) \text { is } \mathrm{SAI} \Longrightarrow A(G) \text { has } \mathrm{PWF} \text {. }
$$

In particular, we have

(6) $L_{1}(G)$ has PWF for all non-discrete locally compact abelian groups $G$,

since $L_{1}(G)$ is always SAI.

\section{Examples of Arens irregular Banach algebras}

In this section, we construct a number of commutative Banach algebras which have various Arens irregularity properties. Most of these Banach algebras are obtained from some Fourier algebras whose Arens irregularity properties are known.

Let $A$ and $B$ be commutative Banach algebras and let $C=A \oplus_{1} B$. It can be seen that

$$
\begin{gathered}
C^{*}=A^{*} \oplus_{\infty} B^{*}, W A P(C)=W A P(A) \oplus_{\infty} W A P(B), \\
\left\langle C^{*} C\right\rangle=\left\langle A^{*} A\right\rangle \oplus_{\infty}\left\langle B^{*} B\right\rangle,\left\langle C^{*} C^{* *}\right\rangle=\left\langle A^{*} A^{* *}\right\rangle \oplus_{\infty}\left\langle B^{*} B^{* *}\right\rangle, \\
C^{* *}=A^{* *} \oplus_{1} B^{* *}, \text { and } Z\left(C^{* *}\right)=Z\left(A^{* *}\right) \oplus_{1} Z\left(B^{* *}\right) .
\end{gathered}
$$

It can also be seen that $C$ is Arens regular (respectively, SAI) if and only if both $A$ and $B$ are Arens regular (respectively, SAI), and $C$ has PWF if and only if both $A$ and $B$ have WF and at least one of $A$ and $B$ has PWF. These facts will be frequently used in what follows.

For convenience, we record below some known results which will be used throughout this section.

Theorem 2. Let $G$ be a locally compact group.

(I) $L_{1}(G)$ is $S A I$.

(II) If $A(G)$ is Arens regular, then $G$ is discrete.

(III) If $G$ is $\sigma$-compact and non-discrete, then $A(G)$ is ENAR.

(IV) $A(S U(3))$ is not $S A I$.

The reader is referred to Lau-Losert [19], Forrest [7], Granirer [11] and Hu [12, and Losert [25], respectively, for Theorem 2(I) - (IV) and related results.

The Banach algebra given below will play an important role in the constructions of examples. We define it as follows: 
Definition 3. Let $l_{2}\left(2^{\mathbb{R}}\right)$ be the usual Banach space with pointwise multiplication. Let $\mathcal{Y}$ be the unitization of $l_{2}\left(2^{\mathbb{R}}\right)$. Then $\mathcal{Y}$ is a unital commutative Banach algebra and hence $\mathcal{Y}$ has WF but not PWF. Since $\mathcal{Y}$ is reflexive, $\mathcal{Y}$ is both Arens regular and SAI. In particular, $W A P(\mathcal{Y})=\mathcal{Y}^{*}$. Also, we have $\left|\mathcal{Y}^{*}\right| \geq\left|2^{\mathbb{R}}\right|>2^{\aleph_{0}}$.

We start with some examples of unital commutative Banach algebras showing all possible combinations of Arens irregularities.

Theorem 4. There exist unital Arens irregular commutative Banach algebras $A_{1}$, $B_{1}, C_{1}$, and $D_{1}$ such that

(i) $A_{1}$ is SAI but not ENAR,

(ii) $B_{1}$ is ENAR but not $S A I$,

(iii) $C_{1}$ is neither SAI nor ENAR,

(iv) $D_{1}$ is both SAI and ENAR.

Proof. (i) Let $A_{1}=A(\mathbb{T}) \oplus_{1} \mathcal{Y}$, where $\mathcal{Y}$ is the Banach algebra given in Definition 3 . Then $A_{1}$ is a unital commutative Banach algebra. Since both $A(\mathbb{T})$ and $\mathcal{Y}$ are SAI and $A(\mathbb{T})$ is Arens irregular, $A_{1}$ is SAI but not Arens regular.

Note that $\mathcal{Y}^{*}=W A P(\mathcal{Y}) \subseteq W A P\left(A_{1}\right)$. We have

$$
\left|A_{1}^{*} / W A P\left(A_{1}\right)\right| \leq\left|A_{1}^{*} / \mathcal{Y}^{*}\right|=\left|A(\mathbb{T})^{*}\right|=\left|l_{\infty}(\mathbb{Z})\right|=2^{\aleph_{0}}<\left|\mathcal{Y}^{*}\right| \leq\left|A_{1}^{*}\right|,
$$

i.e., $\left|A_{1}^{*} / W A P\left(A_{1}\right)\right|<\left|A_{1}^{*}\right|$. By the definition of extreme non-Arens regularity, $A_{1}$ cannot be ENAR.

(ii) Let $B_{1}=A(S U(3))$. Then $B_{1}$ is a unital Arens irregular commutative Banach algebra which is ENAR but not SAI (by Theorem 2(II) - (IV)).

(iii) Let $C_{1}=A(S U(3)) \oplus_{1} \mathcal{Y}$, where $\mathcal{Y}$ is the same as above. Then $C_{1}$ is a unital commutative Banach algebra which is neither Arens regular nor SAI, since $A(S U(3))$ is Arens irregular and not SAI.

Since $A(S U(3))$ is separable, $\left|A(S U(3))^{*}\right| \leq 2^{\aleph_{0}}$. By similar arguments as used in the proof of (i), we have $\left|C_{1}^{*} / W A P\left(C_{1}\right)\right| \leq\left|A(S U(3))^{*}\right| \leq 2^{\aleph_{0}}<\left|\mathcal{Y}^{*}\right| \leq\left|C_{1}^{*}\right|$. Therefore, $C_{1}$ is not ENAR.

(iv) Let $D_{1}=A(\mathbb{T})$. Then $D_{2}$ is a unital Arens irregular commutative Banach algebra which is both SAI and ENAR (by Theorem 2(I) - (III)).

For any locally compact group $G, W A P(\widehat{G})$ will denote $W A P(A(G))$. When $G$ is abelian with dual group $\widehat{G}, W A P(A(G))$ is precisely the space of weakly almost periodic functions on $\widehat{G}$.

By $(6), L_{1}(\mathbb{R})$ has PWF. Like $A(\mathbb{T}), L_{1}(\mathbb{R})(\cong A(\mathbb{R}))$ is both SAI and ENAR (by Theorem $2(\mathrm{I})$ and $(\mathrm{III})$ ). Note that $A(\mathbb{T})$ is unital and $L_{1}(\mathbb{R})$ has a BAI. In the following, we give more examples of commutative Banach algebras with different combinations of Arens irregularities such that each of them has PWF and can be constructed without possessing any BAI.

Theorem 5. There exist commutative Banach algebras $A_{2}, B_{2}, C_{2}$, and $D_{2}$ which have $P W F$ (and hence are Arens irregular) and are such that

(i) $A_{2}$ is SAI but not ENAR,

(ii) $B_{2}$ is ENAR but not $S A I$,

(iii) $C_{2}$ is neither $S A I$ nor ENAR,

(iv) $D_{2}$ is both SAI and ENAR.

Moreover, such Banach algebras $A_{2}, B_{2}, C_{2}$, and $D_{2}$ can be constructed with or without a BAI. 
Proof. (i) Let $A_{2}=L_{1}(\mathbb{T}) \oplus_{1} \mathcal{Y}$, where $\mathcal{Y}$ is the Banach algebra defined in Definition 3. A similar argument as used in the proof of Theorem 4(i) shows that $A_{2}$ is SAI but not ENAR. Clearly, both $L_{1}(\mathbb{T})$ and $\mathcal{Y}$ have WF. By $(6), L_{1}(\mathbb{T})$ has PWF. Therefore, $A_{2}=L_{1}(\mathbb{T}) \oplus_{1} \mathcal{Y}$ has PWF.

(ii) Let $G$ be a locally compact abelian group satisfying $\chi(G)>\kappa(G)=\aleph_{0}$ (e.g., take $G$ to be the product of $\mathbb{Z}$ and a non-metrizable compact abelian group). Let $B_{2}=A(S U(3)) \oplus_{1} A(G)$. Then $B_{2}$ is not SAI because $A(S U(3))$ is not SAI.

However, since $A(S U(3))$ is unital and $A(G)$ is SAI, they both have WF. By (5), $A(G)$ has PWF. Therefore, $B_{2}=A(S U(3)) \oplus_{1} A(G)$ has PWF.

We show below that $B_{2}$ is ENAR. For a Banach space $M$, let $\mathcal{D}(M)$ denote the density character of $M$. By [14, Theorem A3], we have

$$
\mathcal{D}\left(B_{2}\right)=\max \{\mathcal{D}(A(S U(3))), \mathcal{D}(A(G))\}=\max \left\{\aleph_{0}, \chi(G)\right\}=\chi(G) .
$$

Note that $B_{2}^{*}=A(S U(3))^{*} \oplus_{\infty} A(G)^{*}$ and $W A P\left(B_{2}\right)=W A P(\widehat{S U(3)}) \oplus_{\infty} W A P(\widehat{G})$. It can be seen that $A(G)^{*} / W A P(\widehat{G})$ is the image of $B_{2}^{*} / W A P\left(B_{2}\right)$ under the linear contraction $B_{2}^{*} / W A P\left(B_{2}\right) \longrightarrow A(G)^{*} / W A P(\widehat{G}),(S, T)+W A P\left(B_{2}\right) \longmapsto T+$ $W A P(\widehat{G})$. By [12, Theorem 4.1] (the case $J=\{0\}$ ) and its proof, we see that there exists a bounded linear map from $A(G)^{*} / W A P(\widehat{G})$ onto $l_{\infty}(\chi(G))$. Thus, there exists a bounded linear map from $B_{2}^{*} / W A P\left(B_{2}\right)$ onto $l_{\infty}\left(\mathcal{D}\left(B_{2}\right)\right)\left(=l_{\infty}(\chi(G))\right)$. It follows from [12, Lemma 2.1] that $B_{2}$ is ENAR.

(iii) Let $C_{2}=A(S U(3)) \oplus_{1} L_{1}(\mathbb{R}) \oplus_{1} \mathcal{Y}$, where $\mathcal{Y}$ is the same as above. Then $C_{2}$ is not SAI because $A(S U(3))$ is not SAI. Since both $A(S U(3))$ and $L_{1}(\mathbb{R})$ are separable, $\left|A(S U(3))^{*} \oplus_{\infty} L_{1}(\mathbb{R})^{*}\right| \leq 2^{\aleph_{0}}$. Following the proof of Theorem 4(i), we have

$$
\left|C_{2}^{*} / W A P\left(C_{2}\right)\right| \leq\left|A(S U(3))^{*} \oplus_{\infty} L_{1}(\mathbb{R})^{*}\right| \leq 2^{\aleph_{0}}<\left|\mathcal{Y}^{*}\right| \leq\left|C_{2}^{*}\right|,
$$

and thus $C_{2}$ is not ENAR.

Since $A(S U(3))$ is unital and $L_{1}(\mathbb{R}) \oplus_{1} \mathcal{Y}$ is SAI, both $A(S U(3))$ and $L_{1}(\mathbb{R}) \oplus_{1} \mathcal{Y}$ have WF. Note that $L_{1}(\mathbb{R})$ has PWF due to (6). Therefore, $C_{2}=A(S U(3)) \oplus_{1}$ $L_{1}(\mathbb{R}) \oplus_{1} \mathcal{Y}$ has PWF.

(iv) Let $D_{2}=A(G)$, where $G$ is the group as in (ii) above. Then $D_{2}$ is both SAI and ENAR and hence, by (5), $D_{2}$ has PWF.

Clearly, each of $A_{2}, B_{2}, C_{2}$, and $D_{2}$ constructed above has a BAI.

The last assertion of the theorem follows if we replace the Banach algebras constructed above by $A_{2} \oplus_{1} l_{2}, B_{2} \oplus_{1} l_{2}, C_{2} \oplus_{1} l_{2}$, and $D_{2} \oplus_{1} l_{2}$, respectively, where the Banach algebra $l_{2}$ with pointwise multiplication does not have any BAI.

Let $A$ be a commutative Banach algebra. Recall assertions (1) and (2) from Section 2. We see that if

$$
\left\langle A^{*} A\right\rangle \varsubsetneqq\left\langle A^{*} A^{* *}\right\rangle \varsubsetneqq A^{*}
$$

then $A$ satisfies

$$
A \varsubsetneqq Z\left(A^{* *}\right) \varsubsetneqq A^{* *}
$$

and $A$ does not have PWF.

We shall give two more examples below showing that commutative Banach algebras can be constructed to satisfy (7) (and hence not being SAI or having PWF) with or without being ENAR. 
Theorem 6. There exist commutative Banach algebras $A_{3}$ and $B_{3}$ which satisfy (7) and are such that

(i) $A_{3}$ is ENAR;

(ii) $B_{3}$ is not ENAR.

Proof. (i) Let $G$ be a non-metrizable compact group, and let $A_{3}=A\left(F_{2}\right) \oplus_{1} A(G)$. Then $A_{3}$ is a commutative Banach algebra which satisfies $(7)$, since so does $A\left(F_{2}\right)$. Following the argument of the last paragraph in the proof of Theorem 5(ii), one sees that $A_{3}$ is ENAR.

(ii) Let $B_{3}=A\left(F_{2}\right) \oplus_{1} \mathcal{Y}$, where $\mathcal{Y}$ is the Banach algebra given in Definition 3. Then $B_{3}$ satisfies condition (7). By the arguments used in the proof of Theorem 4(i) again, one can derive that $B_{3}$ is not ENAR.

We recall that (7) holds for $A\left(F_{2}\right)$ (see Section 2). However, the Banach algebras $B_{1}, C_{1}, B_{2}$ and $C_{2}$ in Theorems 4 and 5 illustrate that, in general, we do not have $(8) \Longrightarrow(7)$. In [5, Chapter 9], Dales and Lau constructed numerous convolution Beurling algebras $l_{1}(G, \omega)$ over discrete abelian groups satisfying (8). We note that these Beurling algebras are unital, and hence (7) fails automatically. The reader is referred to [5] for some natural examples of non-commutative Banach algebras $A$ whose two topological centres are strictly between $A$ and $A^{* *}$.

Due to the fact that $A\left(F_{2}\right)$ satisfies (7), the following question was raised by the first author in [15]:

Problem 1. Does $\left\langle A(G)^{*} A(G)^{* *}\right\rangle=A(G)^{*}$ imply that $G$ is amenable?

By (2), a positive answer to Problem 1 would also give a positive answer to the following

Problem 2. Does strong Arens irregularity of $A(G)$ imply that $G$ is amenable?

It is known that a locally compact group $G$ is amenable if and only if $A(G)$ has a BAI (see Leptin [24]). Our Theorem 5 shows that there do exist commutative Banach algebras closely related to Fourier algebras which are SAI and have PWF but do not have any BAI. While we do not know the answers to Problems 1 and 2, this seems to indicate that they are both negative.

In [20, Remark 6.6(a)], Lau and Losert asked whether $\left\langle A(G)^{*} B_{\rho}(G)\right\rangle=A(G)^{*}$ is true when $G$ is not amenable, where $B_{\rho}(G)$ is the reduced Fourier-Stieltjes algebra of $G$. Note that $\left\langle A(G)^{*} B_{\rho}(G)\right\rangle \subseteq\left\langle A(G)^{*} A(G)^{* *}\right\rangle$ and the equality holds when $G$ is discrete (see $\mathrm{Hu}$ [15. Corollary 3.1]). As shown by Losert [24, $F_{2}$ gives an example of a non-amenable group $G$ for which $A(G)^{*}$ does not factor over $B_{\rho}(G)$. This leads naturally to the question below, which coincides with Problem 1 when $G$ is discrete.

Problem 3. If $A(G)^{*}$ factors over $B_{\rho}(G)$, is $G$ amenable?

Note that for all discrete groups $G$,

$$
A(G) \oplus\left\langle A(G)^{*} B_{\rho}(G)\right\rangle^{\perp} \subseteq Z\left(A(G)^{* *}\right)
$$

by [15, Corollary 3.1]. Comparing with Ülger [28, Theorem 3.3(e)] for the Fourier algebra case (cf. (4)), we may consider the following.

Problem 4. For a (non-amenable) discrete group $G$, if $A(G)^{*}$ factors over $B_{\rho}(G)$, is $A(G) \mathrm{SAI}$ ? 
We note that an affirmative answer to Problem 1 would also yield affirmative answers to Problems 3 and 4. The reader is referred to Lau-Losert [21], Losert [24, and Ülger [28] for more discussions on problems relating to topological centres of Fourier algebras.

We feel it is still a very interesting question to exhibit a general class of Banach algebras (containing such natural Banach algebras as $L_{1}(\mathbb{R})$ ) which are both SAI and ENAR.

Let $H^{1}(\mathbb{T})=\left\{f \in L_{1}(\mathbb{T}): \widehat{f}(-n)=0\right.$ for all $\left.n \in \mathbb{N}\right\}$. Then $H^{1}(\mathbb{T})$ is a closed ideal in $L_{1}(\mathbb{T})$. Among other results proved by Ülger 29 on Riesz sets in locally compact abelian groups, 29, Corollary 2.5] shows that $H^{1}(\mathbb{T})$ is Arens regular. This result was also proved by Graham in 8. This example tells us in particular that an SAI and Arens irregular Banach algebra can have an infinite dimensional closed ideal which is Arens regular. See also Auger [2, Theorem 4.1.1] for Banach algebras with this property.

We conclude this paper by showing that it is possible for a commutative Banach algebra to contain an infinite dimensional Arens regular closed ideal while possessing all three Arens irregularity properties as considered in the present paper.

Proposition 7. There exists an ENAR and SAI commutative Banach algebra A with PWF such that $A$ has an infinite dimensional closed ideal which is Arens regular.

Proof. Let $G$ be a locally compact abelian group satisfying $\chi(G)>\kappa(G)=\aleph_{0}$. Let $A=A(G) \oplus_{1} l_{2}$. Then $A$ is both ENAR and SAI, and it has PWF (see the proof of Theorem 5). Clearly, $l_{2}$ is an infinite dimensional closed ideal in $A$ and is Arens regular.

\section{REFERENCES}

1. R. Arens, The adjoint of a bilinear operation, Proc. Amer. Math. Soc. 2 (1951), 839-848. MR0045941(13:659f)

2. C. Auger, Jordan Arens irregularity, Master's thesis, Carleton University, 2007.

3. A. Bouziad and M. Filali, On the size of quotients of function spaces on a topological group, preprint (2008).

4. H. G. Dales, Banach Algebras and Automatic Continuity, London Math. Society Monographs, New Series 24, Oxford University Press, New York, 2000. MR.1816726 (2002e:46001)

5. H. G. Dales and A. T.-M. Lau, The second duals of Beurling algebras, Mem. Amer. Math. Soc. 177 (2005), no. 836. MR2155972 (2006k:43002)

6. C. K. Fong and M. Neufang, On the quotient space $U C(G) / W A P(G)$ and extreme non-Arens regularity of $L_{1}(H)$, preprint (2006).

7. B. Forrest, Arens regularity and discrete groups, Pacific J. Math. 151 (1991), 217-227. MR $1132386(93 \mathrm{c}: 43001)$

8. C. C. Graham, Arens regularity of $H^{1}$, preprint.

9. E. E. Granirer, Weakly almost periodic and uniformly continuous functionals on the Fourier algebra of any locally compact group, Trans. Amer. Math. Soc. 189 (1974), 371-382. MR 0336241 (49:1017)

10. E. E. Granirer, On group representations whose $C^{*}$-algebra is an ideal in its von Neumann algebra, Ann. Inst. Fourier (Grenoble) 29 (1979), 37-52. MR558587 (81b:22007)

11. E. E. Granirer, Day points for quotients of the Fourier algebra $A(G)$, extreme nonergodicity of their duals and extreme non-Arens regularity, Illinois J. Math. 40 (1996), 402-419. MR:1407625 (98c:43005)

12. Z. Hu, Extreme non-Arens regularity of quotients of the Fourier algebra $A(G)$, Colloq. Math. 72 (1997), 237-249. MR1426699 (98a:43004)

13. Z. Hu, Inductive extreme non-Arens regularity of the Fourier algebra $A(G)$, Studia Math. 151 (2002), 247-264. MR1917836 (2003f:46069) 
14. Z. Hu, Maximally decomposable von Neumann algebras on locally compact groups and duality, Houston J. Math. 31 (2005), 857-881. MR2148807 (2006e:22010)

15. Z. Hu, Open subgroups and the centre problem for the Fourier algebra, Proc. Amer. Math. Soc. 134 (2006), 3085-3095. MR2231636 (2007e:22004)

16. Z. Hu and M. Neufang, Decomposability of von Neumann algebras and the Mazur property of higher level, Canad. J. Math. 58 (2006), 768-795. MR2245273(2008e:46076)

17. Z. Hu, M. Neufang, and Z.-J. Ruan, Multipliers on a new class of Banach algebras, locally compact quantum groups, and topological centres, preprint.

18. Z. Hu, M. Neufang, and Z.-J. Ruan, On topological centre problems and SIN-quantum groups, preprint.

19. A. T.-M. Lau and V. Losert, On the second conjugate algebra of $L_{1}(G)$ of a locally compact group, J. London Math. Soc. (2) 37 (1988), 464-470. MR939122 (89e:43007)

20. A. T.-M. Lau and V. Losert, The $C^{*}$-algebra generated by operators with compact support on a locally compact group, J. Funct. Anal. 112 (1993), 1-30. MR.1207935 (94d:22005)

21. A. T.-M. Lau and V. Losert, The centre of the second conjugate algebra of the Fourier algebra for infinite products of groups, Math. Proc. Cambridge Philos. Soc. 138 (2005), 27-39. MR2127225 (2006c:43003)

22. A. T.-M. Lau and A. Ülger, Topological centers of certain dual algebras, Trans. Amer. Math. Soc. 348 (1996), 1191-1212. MR.1322952 (96h:43003)

23. H. Leptin, Sur l'algèbre de Fourier d'un groupe localement compact, C. R. Acad. Sci. Paris, Sér. A-B 266 (1968), A1180-A1182. MR0239002 (39:362)

24. V. Losert, The centre of the bidual of Fourier algebras, preprint.

25. V. Losert, On the centre of the bidual of Fourier algebras (the compact case), presentation at the 2004 Istanbul International Conference on Abstract Harmonic Analysis.

26. T. W. Palmer, Banach Algebras and the General Theory of *-Algebras, Volume 1, Cambridge University Press, Cambridge, 1994. MR.1270014 (95c:46002)

27. J. S. Pym. The convolution of functionals on spaces of bounded functions, Proc. London Math. Soc. 15 (1965), 84-104. MR0173152(30:3367)

28. A. Ülger, Central elements of $A^{* *}$ for certain Banach algebras A without bounded approximate identities, Glasg. Math. J. 41 (1999), 369-377. MR1720442 (2001b:46082)

29. A. Ülger, Characterizations of the Riesz sets, preprint.

Department of Mathematics and Statistics, University of Windsor, Windsor, Ontario, N9B 3P4, CANADA

E-mail address: zhiguohu@uwindsor.ca

School of Mathematics and Statistics, Carleton University, Ottawa, Ontario, K1S 5B6, CANADA

E-mail address: mneufang@math.carleton.ca 